Instituto Internacional de Investigación y Desarrollo Tecnológico Educativo INDTEC, C.A.

DOI: https://doi.org/10.29394/Scientific.issn.2542-2987.2019.4.13.8.160-181

OAl-PMH: http://www.indteca.com/ojs/index.php/Revista Scientific/oai

Artículo Original / Original Article

\title{
Incidencia del Desempeño Profesional del Docente de Educación Inicial
}

Autores: Mónica Alexandra Salto Cubillos Universidad Nacional de Educación, UNAE monica75salto@gmail.com

Cuenca, Ecuador https://orcid.org/0000-0003-3583-7196

Adrián Eduardo Cendón Sosa Movimiento Escultista Ecuatoriano, SCOUT adricendon@gmail.com Cuenca, Ecuador https://orcid.org/0000-0002-9467-7033

\section{Resumen}

El presente trabajo versa sobre la Evaluación del Desempeño Profesional del Docente de Educación Inicial del Circuito 03-04-13 de la ciudad de Cuenca, período 2018; el objetivo de la investigación fue determinar el desempeño profesional del docente de Educación Inicial "Ciudad de Cuenca", período 2018, mediante un estudio descriptivo, con el propósito de establecer la calidad de la educación impartida. Participaron en la investigación 14 docentes del nivel, 76 padres de familia, a los cuales se les aplico la encuesta. Por otra parte, se procedió a realizar la investigación del fundamento científico, vinculado a la calidad educativa; lo que favoreció en ampliar el conocimiento del tema investigado. Finalmente, la triangulación entre la revisión bibliográfica, la experiencia de los autores y los resultados de la recolección de datos, emitiendo conclusiones del desempeño profesional del docente de nivel inicial en el CEI "Ciudad de Cuenca" la calidad educativa es nivel 3, es decir Satisfactorio, asunto sobre el cual se realizó una propuesta de formación.

Palabras clave: evaluación; profesional; docente.

Fecha de Recepción: 27-02-2019
Fecha de Aceptación: 19-06-2019
Fecha de Publicación: 05-08-2019 


\title{
Incidence of the Professional Performance of the Teacher of Initial Education
}

\begin{abstract}
The present work focuses on: evaluation of the teaching professional performance of an initial education center in circuit 03-04-13 of Cuenca city, period 2018; the objective of the research was to determine teaching professional performance of initial education "Ciudad de Cuenca", period 2018, Center through a descriptive study, with the purpose of establishing the quality of the education provided. They participated in the research level 14 teachers, 76 parents, to which the survey was applied. On the other hand, the investigation of the scientific basis, linked to educational quality, was carried out; what favored in expanding the knowledge of the subject investigated. Finally, the triangulation between the bibliographic review, the experience of the authors and the results of the data collection, issuing conclusions of the professional performance of the teacher of initial level in the IEC "Ciudad de Cuenca" the quality of education is level 3, equals to satisfactory, subject on which a educating proposal was made.
\end{abstract}

Keywords: evaluation; professional; teacher.

Date Received: 27-02-2019
Date Acceptance:

19-06-2019
Date Publication:

05-08-2019 


\section{Introducción}

Para Ministerio de Educación (2017a), el desempeño profesional docente, hace referencia a: "descripciones de lo que debe hacer un profesional educativo competente; es decir, de las prácticas que tienen una mayor correlación positiva con la formación que se desea que los estudiantes alcancen" (pág. 7); por ello, la evaluación del desempeño profesional del docente de Educación Inicial, tiene como propósito identificar el desempeño profesional frente al trabajo con niños(as) de entre 3-5 años de edad psicológicamente considerados del aprendizaje preoperacional, según Castilla (2014): quien menciona las etapas cognitivas de Piaget (pág. 20).

La problemática parte por el desconocimiento de los estándares de calidad, lo cual conlleva a un desempeño profesional docente monótono y automatizado. También se debe mencionar la falta de compromiso ético profesional lo cual pone de manifiesto en el docente una irresponsabilidad en sus actividades profesionales, y el escaso desarrollo de destrezas en el aprendizaje, por lo que se presentan niños con bajo desarrollo integral. De esta forma, no se orienta al estudiante a conseguir las destrezas, incumpliendo los estándares de calidad del desempeño profesional docente que velan por aprendizajes con calidad y calidez.

Lo indicado, permite comprender el desarrollo integral del niño, puesto que en el nivel inicial los niños desarrollan todas sus habilidades de forma espiral, los ámbitos de aprendizaje, por lo que es necesario que las destrezas sean adquiridas a través de juegos y mediante la interrelación del niño con su entorno y pares sociales. Por otra parte, Ovalles (2018), expresa que:

Los docentes de educación inicial necesitan aprender a identificar oportunamente cuáles son las distintas capacidades y potencialidades de cada uno de los niños a su cargo, y así aplicar estrategias de trabajo diseñadas especialmente para proporcionar el pleno desarrollo de sus capacidades y potencialidades (pág. 26). 
La importancia de esta investigación, radicó en que sirvió de base en cuanto a los datos recolectados y analizados por ser el primer trabajo que se refirió al desempeño profesional del docente de nivel inicial, para los docentes y la sociedad, a más del profesionalismo individual.

Para determinar los resultados del estudio del problema planteado se aplicaron técnicas investigativas y sus correspondientes instrumentos de recolección de datos con ítems en las encuestas elaborados por la autora y validados por experto.

En evaluación del desempeño profesional del docente, se llevó a cabo la hetero-evaluación (padres-docente), la coevaluación (directivos-docentes), y autoevaluación (reflexión propia), apegados a los indicadores de los estándares de calidad del año 2017, siendo una de las motivaciones principales propender a la mejora de la calidad educativa, se trabajó con una muestra de 14 docentes, 76 padres de familia, y sin estudiantes por la edad del grupo y la subjetividad en sus respuestas.

El objetivo general del presente trabajo investigativo, fue determinar el desempeño profesional docente del Educación Inicial del Centro de El "Ciudad de Cuenca", período 2018, mediante un estudio descriptivo, con el propósito de establecer la calidad de la educación impartida. De otro lado, los objetivos específicos fueron: Analizar los datos recogidos en los test aplicados para interpretar los resultados; determinar el desempeño profesional docente de la en base al cumplimiento de los estándares de calidad en los procesos de enseñanza-aprendizaje; y establecer la calidad de la educación impartida en por los docentes de Educación Inicial, período 2018.

La hipótesis planteada para la presente investigación fue: Los docentes de Educación Inicial período 2018, desempeñan su labor docente para la adquisición de aprendizajes con calidad y calidez según los Estándares de Calidad Nacional. 


\section{Marco Teórico}

\subsection{La Educación Inicial}

La educación inicial, es un período en el que la acción educativa está centrada en actividades que responden a los intereses de los niños y niñas, contribuyendo directamente al desarrollo de su expresión personal dentro de su entorno escolar.

Al basarse los aprendizajes en experiencias del niño y sus curiosidades para conocer el mundo que le rodea, el contacto con la naturaleza ayuda mucho para que el docente oriente con mayor facilidad la significatividad de lo vivido, además de que posibilita compartir con sus pares, la experiencia, que sirve de guía para que el profesional docente encamine las distintas actividades a aprendizajes de calidad.

La educación inicial, para el Ministerio de Educación (2014): está dentro de las políticas públicas como una prioridad del desarrollo integral del niño, siendo un desafío desde el fortalecer las estrategias para dicho desarrollo temprano, pues en estas etapas se da el desarrollo fututo del niño como ser y persona (pág. 7).

Como un aporte valioso, es el hecho de que en la educación el niño ha de permanecer en su naturaleza. Por ende, Villarroel (2015): expone que la escolaridad debe ser gradual interviniendo lo menos posible en su proceso de formación (pág. 7). En consecuencia, se debe empezar con la edad corta, de tal forma que la escolaridad se convierta en un proceso natural y parte de su desarrollo individual y social.

Según Dones (2016): que es precursor en analizar las perspectivas de María Montessori, destaca que la educación a edades tempranas, debe ser por percepción ya que se está en una etapa de gran receptividad sensorial. La "mente absorbente" fue la forma como se reconoció el potencial de los niños más pequeños, al sostener que los mismos se encontraban transitando un período embrionario junto con la gestación espiritual. Así, se justifica la 
importancia de la educación inicial (pág. 7).

Se comprende entonces que la educación inicial es un tiempo para recrear experiencias y actividades lo más cercanas a la realidad cotidiana del niño, proporcionando a éste el medio para que estas vivencias, le permitan adquirir dominios y habilidades propios de la edad, suficientes y significativos para que enfrente sus pequeños problemas, con sus pares, y dentro del entorno que lo rodea.

\subsection{La buena práctica profesional docente en el nivel inicial}

Una buena práctica profesional del docente del nivel inicial según Escobar (2014a): está en la motivación en el proceso de enseñanzaaprendizaje, en la flexibilidad pedagógica; en el logro de la percepción en los estudiantes respecto a sus propios aprendizajes; en su desenvolvimiento en el contexto social; en la comunicación entre docente-dicente; y en la inactividad o interacción dentro del aula (págs. 4-8).

El Ministerio de Educación Nacional (2013), señala que: la incidencia de una buena práctica del profesor del nivel inicial, ocurre cuando se parte del desarrollo y aplicación de proyectos áulicos que parten del interés del niño, se recrea rincones para favorecer experiencias significativas, se aplica estrategias didácticas activas, innovadoras, investigativas, con intencionalidad de enseñanza, además de que en los ambientes generen la creatividad e imaginación del niño, se propone juegos como forma de aprender; se favorecen actividades que motiven el desarrollo motor grueso y fino; y se recrea situaciones que fomentan habilidades socio-afectivas (pág. 27).

Según Soriano y Delgado (2011): el docente del mencionado nivel inicia, influye en "la capacidad de interaccionar con los alumnos, en la adaptación a diferentes situaciones, la motivación, la organización, y/o la corrección de errores, empatía con los mismos, en el Ser-Saber estar y en la capacidad de estar abierto a aprender" (págs. 9-10). 
Partiendo de la experiencia misma, la incidencia de la buena práctica docente en el nivel inicial es fundamental para el niño dentro de su proceso de desarrollo ya que, al ser su primera experiencia escolarizada, el docente debe cuidar que sea una experiencia alentadora, ilusionada, propendiéndose a que este niño forme una conducta de aprender de forma intrínseca. Vale recordar que el nivel inicial no solo desarrolla habilidades, sino que es el primer punto de desapegue por lo que crea un equilibro entre su seguridad, confianza y su autonomía frente a sus progenitores.

Por todo lo antes indicado, el docente del nivel inicial es la motivación y el enamoramiento primero del infante para amar al aprendizaje, y forjar en el ese placer por aprender, pues nace de la ejemplificación y la actitud que el docente promueve en los estudiantes, antes, durante y después de un proceso de enseñanza.

\subsection{Evaluación de Desempeño Profesional del Docente de Nivel Inicial}

La visión general de la evaluación se enfoca a reconocer el valor de algo, en este caso de la docencia, refiriéndose a las acciones que el docente realiza en bien de brindar un servicio de calidad. Para Valdés (2000), citado por Tejedor (2012a): "afirma que la evaluación del desempeño docente es una actividad de análisis, compromiso y formación del profesorado, que valora y enjuicia la concepción, práctica, proyección y desarrollo de la actividad y de la profesionalización docente" (pág. 321). Es que el análisis de un trabajo diario, pero con diferentes personalidades y temperamentos cambiantes debe generar un compromiso de responsabilidad pedagógica y formación constante, pues el ser humano es un ser perfectible mas no perfecto, que en gran parte es guiado en esa perfección por el docente.

El Ministerio de Educación (2017b), indica que la evaluación del desempeño docente es: "el conjunto de acciones organizadas de acuerdo con las mediaciones e interacciones pedagógicas entre el conocimiento científico 
y el conocimiento escolar, y con las mediaciones socioculturales y lingüísticas" (pág. 1).

No solo se puede evaluar un conocimiento cognitivo amplio que siendo importante no es el único que debe manejar un profesional de la educación, pues su calidad de orientador y facilitador de experiencias para aprendizajes debe favorecerle con una visión holística del ser, con su vivencialidad diaria, con los valores con los que se desempeñe, además de la convivencia diaria, los que son básicos para ejemplificar y ejecutar un desempeño eficiente y eficaz.

Finalmente, Hernández (2017): expresa que toda evaluación incluida la del docente debe estar dentro del marco del modelo educativo, para retroalimentar y en una interacción entre la enseñanza y el currículo nacional que se pretende ejecutar juntamente con la capacitación y conocimientos de los maestros. Así, este ejercicio tendrá un impacto positivo en el desarrollo del profesor y en la calidad de educación que los estudiantes recibirán (pág. 6).

Se debe considerar que la evaluación del desempeño profesional docente a través de una reflexión propia y una visión de los otros actores de la educación, busca siempre la mejora de su ejercicio profesional en bien de que éste oriente y facilite aprendizajes de calidad, donde el estudiante salga siempre beneficiados, pues la mejora del profesional en ámbitos académicos, éticos, sociales, y culturales terminarán aplicados como medios para el fin único de la enseñanza.

Para la evaluación del desempeño profesional docente en el Ecuador se presentaron los estándares de calidad que contienen criterios generales clasificados en componentes, subdivididos en procesos y en indicadores de acuerdo al actor educativo que los realiza. 


\subsection{Importancia e influencia del desempeño profesional docente}

El rol del docente es muy importante e influyente a la hora de la práctica profesional, pues debe ser el primero en aplicar todas las actualizaciones, y capacitaciones que realice, considerando que los contextos son diferentes, pero existe siempre la necesidad de brindar calidad educativa mediante el excelente desempeño profesional docente.

La importancia radica en que el desempeño profesional docente permite orientar y fortalecer procesos de enseñanza-aprendizaje. Para Arratia (2010): "relación entre el docente y el desarrollo de los aprendizajes de los alumnos, categorizando y estandarizando determinadas conductas docentes" (pág. 18); además favorece la proyección a futuro, y retroalimentar efectivamente a los docentes con menor desempeño.

Por otro lado, Espinoza (2014): considera que la importancia e influencia del desempeño profesional docente, se presenta en factores como la formación inicial y capacitación docente, la motivación, la relación profesorestudiante y familia-institución, organización institucional, políticas educativas, perfil docente, los dominios que debe tener cognitivos, emocionales, psíquicos y éticos, el desarrollo profesional y preparación para los desafíos docentes, y guiar la relación familia, institución y comunidad (pág. 6).

Además, concordando con el Proyecto Regional de Educación para América Latina y el Caribe (PRELAC), Robalino (2015): presenta que el desempeño profesional del docente es el foco central de los aprendizajes, ya que siendo uno de los actores educativos y al darse la interrelación docentedicente, reúne en un solo punto las condiciones, espacios, bases pedagógicas y didácticas, la mediación y la intención pedagógica, formando una espiralidad formativa para los aprendizajes significativos (pág. 12).

Se puede decir entonces, que la importancia del desempeño profesional docente radica en la proyección de una entrega completa del docente como humano, y profesional, que guía sus acciones a un solo objetivo que es brindar 
la calidad en los aprendizajes.

\section{Marco Metodológico}

\subsection{Diseño de investigación}

El tipo de estudio fue de corte cuali-cuantitativo, o llamado también mixto, no experimental. El tipo de investigación fue descriptivo porque sirvió para describir, desde varios enfoques, el desempeño profesional de los docentes del Centro de Educación Inicial "Ciudad de Cuenca", en el período 2018.

Se trabajó con una muestra de 76 padres de familia y 14 docentes de los cuales 13 son del sexo femenino y 1 del sexo masculino. En el caso de los niños, por ser de la edad entre 3-5 años, y no tener una mirada objetivo, no se les consideró.

\subsubsection{Métodos}

El método que guio la investigación fue el Método Científico, con sus modalidades Inductivas y Deductivas.

\subsubsection{Técnicas}

a). Encuesta: Se aplicó la encuesta a 76 padres de familia de la institución educativa en la que se realizó la investigación. Para ello, se empleó el respectivo instrumento validado.

b). Entrevista: La entrevista se la llevó a cabo con cada docente en base del instrumento de autoevaluación.

\subsubsection{Procedimientos}

Se obtuvo el permiso del Distrito para el estudio en la institución fiscal, de parte de la autoridad del establecimiento; se procedió a firmar el 
consentimiento informado de confidencialidad; se validaron los instrumentos de aplicación, para luego procesarlos y analizarlos.

Se aplicaron 4 instrumentos de evaluación elaborados por la autora, tomando como base los Indicadores de los Estándares de Calidad del año 2017, que fueron validados en muestras pequeñas obteniendo coincidencia en los resultados y la aprobación del experto.

Los instrumentos aplicados permitieron tener una visión holística del desempeño profesional del docente del Nivel Inicial, mediante: la autoevaluación, referido a una reflexión intrínseca del desempeño laboral; la coevaluación, realizada por parte del director hacia los docentes; la heteroevaluación, ejecutada por los padres de familia. Además, se consideraron como indicadores valorativos, los mismos que se plantean en los estándares de calidad. Cada instrumento presentó 5 componentes: de gestión administrativa; gestión pedagógica; convivencia; de servicios educativos; y sistema integral de gestión de riesgos escolares.

La medida estándar que se aplicó fue la de niveles con la siguiente valoración: Nivel 4: Destacado; Nivel 3: Satisfactorio; Nivel 2: En proceso; Nivel 1: Insatisfactorio.

De acuerdo a esto, se pudo determinar el nivel de calidad educativa del desempeño profesional docente que se está prestando institucionalmente.

\subsubsection{Resultados Análisis}

\subsubsection{Coevaluación del Desempeño Profesional del Docente de Nivel Inicial.}

En la Coevaluación del Desempeño Profesional Docente, se consideraron como Componentes: la Gestión Administrativa, Gestión Pedagógica, Convivencia, Servicios Educativos y el Sistema Integral de Gestión de Riesgos Laborales. 
Tabla 1: Desempeño Profesional del Docente de Nivel Inicial: Coevaluación.

\begin{tabular}{|l|c|c|c|c|c|c|c|c|}
\cline { 2 - 9 } \multicolumn{1}{c|}{} & \multicolumn{4}{c|}{ FRECUENCIA } & \multicolumn{4}{c|}{ PORCENTAJE } \\
\cline { 2 - 9 } \multicolumn{1}{c|}{} & $\begin{array}{c}\text { Nivel } \\
\mathbf{1}\end{array}$ & $\begin{array}{c}\text { Nivel } \\
\mathbf{2}\end{array}$ & $\begin{array}{c}\text { Nivel } \\
\mathbf{3}\end{array}$ & $\begin{array}{c}\text { Nivel } \\
\mathbf{4}\end{array}$ & $\begin{array}{c}\text { Nivel } \\
\mathbf{1}\end{array}$ & $\begin{array}{c}\text { Nivel } \\
\mathbf{2}\end{array}$ & $\begin{array}{c}\text { Nivel } \\
\mathbf{3}\end{array}$ & $\begin{array}{c}\text { Nivel } \\
\mathbf{4}\end{array}$ \\
\hline $\begin{array}{l}\text { Componente de Gestión } \\
\text { Administrativa }\end{array}$ & 0 & 0 & 4 & 10 & $0 \%$ & $0 \%$ & $26 \%$ & $\mathbf{7 4 \%}$ \\
\hline $\begin{array}{l}\text { Componente de Gestión } \\
\text { Pedagógica }\end{array}$ & 0 & 1 & 5 & 8 & $0 \%$ & $7 \%$ & $33 \%$ & $\mathbf{5 7 \%}$ \\
\hline $\begin{array}{l}\text { Componente de } \\
\text { Convivencia }\end{array}$ & 0 & 2 & 6 & 6 & $0 \%$ & $14 \%$ & $43 \%$ & $43 \%$ \\
\hline $\begin{array}{l}\text { Componente de Servicios } \\
\text { Educativos }\end{array}$ & 0 & 0 & 6 & 8 & $0 \%$ & $0 \%$ & $43 \%$ & $\mathbf{5 7 \%}$ \\
\hline $\begin{array}{l}\text { Componente del Sistema } \\
\text { Integral de Gestión de } \\
\text { Riesgos Escolares }\end{array}$ & 0 & 0 & 6 & 8 & $0 \%$ & $0 \%$ & $43 \%$ & $\mathbf{5 7 \%}$ \\
\hline \multicolumn{2}{|c|}{ PROMEDIO } & & & & $4 \%$ & $38 \%$ & $\mathbf{5 8 \%}$ \\
\hline
\end{tabular}

Fuente: Los Autores (2018).

En la coevaluación, la Tabla 1, se considera la visión del directivo para con los docentes a su cargo. Se debe indicar que, en el componente de gestión administrativa, se ha determinado que un $74 \%$ de los docentes se encuentran en el nivel 4 , es decir, Destacado, y un $26 \%$ de docentes, en un nivel satisfactorio. Por otra parte, en la gestión pedagógica, el 57\%, se ubican en el nivel 4; el 33\%, en nivel 3, y el 7\%, en nivel 2, equivalente a En Proceso. Para el componente convivencia, se marcó una igualdad entre el nivel 3 y 4 ; con un $43 \%$ de los docentes, y en el nivel 2; se registraron un $14 \%$ de docentes. En el caso del componente servicios educativos, se registraron $57 \%$ de docentes, en el nivel 4 , pero no muy lejos está el nivel 3 , con el $43 \%$. Finalmente, en el componente de Sistema Integral de Gestión de Riesgos Escolares, se registró un $57 \%$ de docentes, en nivel 4 , y $43 \%$, en nivel 3 .

\subsubsection{Autoevaluación del Desempeño Profesional del Docente de Nivel} Inicial.

En la Autoevaluación del Desempeño Profesional Docente, se contemplaron como Componentes: Gestión Administrativa, Gestión 
Pedagógica, Convivencia, Servicios Educativos y el Sistema Integral de Gestión de Riesgos Laborales.

Tabla 2: Desempeño Profesional del Docente de Nivel Inicial: Autoevaluación.

\begin{tabular}{|l|c|c|c|c|c|c|c|c|}
\cline { 2 - 9 } \multicolumn{1}{c|}{} & \multicolumn{4}{c|}{ FRECUENCIA } & \multicolumn{4}{c|}{ PORCENTAJE } \\
\cline { 2 - 9 } \multicolumn{1}{c|}{} & $\begin{array}{c}\text { Nivel } \\
\mathbf{1}\end{array}$ & $\begin{array}{c}\text { Nivel } \\
\mathbf{2}\end{array}$ & $\begin{array}{c}\text { Nivel } \\
\mathbf{3}\end{array}$ & $\begin{array}{c}\text { Nivel } \\
\mathbf{4}\end{array}$ & $\begin{array}{c}\text { Nivel } \\
\mathbf{1}\end{array}$ & $\begin{array}{c}\text { Nivel } \\
\mathbf{2}\end{array}$ & $\begin{array}{c}\text { Nivel } \\
\mathbf{3}\end{array}$ & $\begin{array}{c}\text { Nivel } \\
\mathbf{4}\end{array}$ \\
\hline $\begin{array}{l}\text { Componente de Gestión } \\
\text { Administrativa }\end{array}$ & 0 & 0 & 5 & 9 & $0 \%$ & $0 \%$ & $36 \%$ & $64 \%$ \\
\hline $\begin{array}{l}\text { Componente de Gestión } \\
\text { Pedagógica }\end{array}$ & 0 & 1 & 5 & 8 & $0 \%$ & $7 \%$ & $36 \%$ & $57 \%$ \\
\hline $\begin{array}{l}\text { Componente de } \\
\text { Convivencia }\end{array}$ & 0 & 1 & 6 & 7 & $0 \%$ & $7 \%$ & $43 \%$ & $50 \%$ \\
\hline $\begin{array}{l}\text { Componente de Servicios } \\
\text { Educativos }\end{array}$ & 0 & 2 & 5 & 7 & $0 \%$ & $14 \%$ & $36 \%$ & $50 \%$ \\
\hline $\begin{array}{l}\text { Componente del Sistema } \\
\text { Integral de Gestión de } \\
\text { Riesgos Escolares }\end{array}$ & 0 & 2 & 4 & 8 & $0 \%$ & $14 \%$ & $29 \%$ & $57 \%$ \\
\hline \multicolumn{2}{|l|}{ PROMEDIO } & & & $0 \%$ & $8 \%$ & $36 \%$ & $56 \%$ \\
\hline
\end{tabular}

Fuente: Los Autores (2018).

Los resultados de la autoevaluación, expresados en la Tabla 2, generan una visión intrínseca de la tarea docente que día a día se realiza. Así, se tiene en el componente gestión administrativa, se detectó que un $64 \%$ de docentes, están en el nivel 4 , mientas que los restantes, esto es el $36 \%$, en el nivel 3 . En gestión pedagógica, un $57 \%$ de docentes, se ubican en el nivel 4 ; un $36 \%$, en nivel 3, y 7\%, en nivel 2. Para el componente convivencia, se detectó que un $50 \%$ de docentes, están en el nivel 4 ; el $43 \%$, en el nivel 3; y el $7 \%$ en el nivel 2. En el componente servicios educativos, se estableció que se ubica un $50 \%$ de los docentes, en el nivel 4; un 36\%, en el nivel 3; y $14 \%$, en el nivel 2. Por último, en el componente Sistema Integral de Gestión de Riesgos Escolares, el $57 \%$ de los docentes, se encuentran en el nivel 4 , un $29 \%$ en el nivel 3 , y un $14 \%$ en el nivel. 


\subsubsection{Heteroevaluación del Desempeño Profesional del Docente de}

Nivel Inicial.

Componentes de la Heteroevaluación del Desempeño Docente, fueron: Gestión Administrativa, Gestión Pedagógica, Convivencia, Servicios Educativos, y el Sistema Integral de Gestión de Riesgos Laborales.

Tabla 3: Desempeño Profesional del Docente de Nivel Inicial: Heteroevaluación.

\begin{tabular}{|c|c|c|c|c|c|c|c|c|}
\hline & \multicolumn{4}{|c|}{ FRECUENCIA } & \multicolumn{4}{|c|}{ PORCENTAJE } \\
\hline & $\begin{array}{c}\text { Nivel } \\
1\end{array}$ & $\begin{array}{c}\text { Nivel } \\
2\end{array}$ & $\begin{array}{c}\text { Nivel } \\
3\end{array}$ & $\begin{array}{c}\text { Nivel } \\
4\end{array}$ & $\begin{array}{c}\text { Nivel } \\
1\end{array}$ & $\begin{array}{c}\text { Nivel } \\
2\end{array}$ & $\begin{array}{c}\text { Nivel } \\
3\end{array}$ & $\begin{array}{c}\text { Nivel } \\
4\end{array}$ \\
\hline $\begin{array}{l}\text { Componente de Gestión } \\
\text { Administrativa }\end{array}$ & 1 & 5 & 28 & 42 & $0 \%$ & $7 \%$ & $38 \%$ & $55 \%$ \\
\hline $\begin{array}{l}\text { Componente de Gestión } \\
\text { Pedagógica }\end{array}$ & 0 & 9 & 33 & 34 & $0 \%$ & $11 \%$ & $43 \%$ & $46 \%$ \\
\hline $\begin{array}{l}\text { Componente de } \\
\text { Convivencia }\end{array}$ & 1 & 3 & 32 & 40 & $0 \%$ & $5 \%$ & $42 \%$ & $53 \%$ \\
\hline $\begin{array}{l}\text { Componente de } \\
\text { Servicios Educativos }\end{array}$ & 1 & 5 & 27 & 43 & $0 \%$ & $8 \%$ & $36 \%$ & $57 \%$ \\
\hline \multirow[t]{2}{*}{$\begin{array}{l}\text { Componente del Sisten } \\
\text { Integral de Gestión de } \\
\text { Riesgos Escolares }\end{array}$} & 1 & 6 & 28 & 41 & $0 \%$ & $9 \%$ & $37 \%$ & $54 \%$ \\
\hline & DEDI & & & & & $8 \%$ & $39 \%$ & $53 \%$ \\
\hline
\end{tabular}

Fuente: Los Autores (2018).

Con una visión externa, en la tabla 3, se tiene la heteroevaluación, la misma que en esta ocasión, se consideraron a los padres de familia para que realicen esta valoración. Así, se tiene que en el componente gestión administrativa, los docentes en un $55 \%$, se ubican en el nivel 4 , destacado, en tanto que los restantes lo hacen: 38\%, en el nivel 3; y 7\%, en el nivel 2. En el componente gestión pedagógica, el $46 \%$ de los docentes, tuvieron un destacado desempeño; los demás se registraron: $43 \%$, en el nivel 3; y $11 \%$, en el nivel 2. Respecto al componente convivencia, los docentes en un 53\%, 
corresponden al nivel 4, un ambiente armónico y convivencia sana; sin embargo, los demás corresponden a: $42 \%$, al nivel 3; y 5\%, al nivel 2. En el componente servicios educativos, el $57 \%$, están en el nivel 4 ; el $36 \%$, se ubican en el nivel 3; y $8 \%$, en el nivel 2 Finalmente, en el componente Sistema Integral de Gestión de Riesgos Escolares, el 54\% de los docentes se ubican en el nivel 4, destacado; los restantes, lo hacen: $37 \%$, en el nivel 3; y $9 \%$, en el nivel 2.

Pero se ve que tan solo con el $53 \%$ de los docentes alcanzan un nivel Destacado, muy seguido de un nivel Satisfactorio con tan solo 39\%. Aquí ya existe un nivel 2, En Proceso, con el 8\%, siendo necesario hacer una pausa puesto que estas son las expresiones de los padres de familia en referencia directa del sentir de sus hijos en el contexto educativo.

\section{Conclusiones}

Luego del análisis de los resultados obtenidos se emite las siguientes conclusiones:

Si se recuerda a Tejedor (2012b): éste mencionaba a la evaluación del desempeño docente como que es una actividad de análisis, compromiso y formación, en la que se valora al personal para proyecciones futuras (pág. 321); así como al Ministerio de Educación (MINEDUC) del Ecuador, que reconoce a la indicada evaluación, como un conjunto de actividades de interacción que obedecen a dimensiones socioculturales, se puede determinar que los docentes, según los componentes de los estándares de calidad del año 2017, están cumpliendo los logros esperados pues en los componentes y sus procesos, ellos comunican, participan, y desarrollan las actividades institucionales, además de involucrarse en la elaboración y aplicación de documentos como el Plan Curricular Institucional y la planificación micro curricular, necesarios para orientar procesos de enseñanza óptimos, sin dejar de lado el apoyo al niño en su retroalimentación In situ, y la guía de los 
servicios educativos como el Departamento de Consejería Estudiantil en las Unidades Distritales de Apoyo a la Inclusión (UDAI).

En cuanto a la convivencia diaria, las actividades de interrelación entre pares son positiva, asertiva, colaborativa, y por tanto, ejemplificadora de una convivencia armónica. Se procura también proteger la integridad del niño ya que éste, según Escobar (2014b): el niño aprende mediante la percepción del contexto social, la comunicación y la actividad o interacción dentro y fuera del aula (págs. 4-8).

Se puede establecer que mantiene un desempeño docente de calidad, que determina que sí se están cumpliendo los estándares de calidad esperados, mismos que promocionan aprendizajes de calidad, significativos en ambientes constructivos de un aprender o aprehender.

Al confrontar la información obtenida en la investigación sobre el desempeño docente, se puede entonces indicar que se confirma la existencia de calidad educativa en los distintos componentes, y tipos de evaluación con un nivel 4, es decir se ha alcanzado un destacado, lo cual lleva a exponer que se logra el objetivo, que proporcionan justamente eficacia y eficiencia en el actuar diario.

En el juicio de valor del Directivo, se pretende reconocer falencias de sus docentes, y a su vez, orientar mejor el trabajo. Los docentes son seres en perfección que necesitan siempre de una mirada externa que les ayude a reconocer falencias, y que se le felicite por los logros. Por ello, es necesaria una propuesta de acción para dar a conocer a los docentes las dificultades encontradas y se lleguen a crear planes de mejora para corregir las falencias en los niveles 3,2 , ya que siempre se propende a la calidad educativa.

Al establecer el desempeño profesional docente se tiene que los docentes están involucrados en sus actividades de forma comprometida con un involucramiento propio. Es por ello, que los promedios del nivel 4 están por encima del $50 \%$ en todos los componentes. Si se considera al desempeño 
como un equilibrio entre las actividades pedagógicas y las institucionales. Esto se verá reflejado en los aprendizajes de los pequeños. Se determina también que las actividades están apegadas a los estándares de calidad educativa.

El contraste con el marco teórico, se muestra que cumpliendo con las directrices que dictamina el Instituto Nacional para la Evaluación de la Educación (INEE, 2017): se impulsa siempre a la mejora e innovación permanente, sin dejar de lado la visión y misión institucional en bien de una educación transcultural, interdisciplinar y multiétnica (pág. 51). Es aquí donde las ventajas de una evaluación al desempeño profesional plasma su objetivo, que de acuerdo a Morán-Barrios (2016): es una clara ventaja por ser una evaluación de $360^{\circ}$ grados; es decir completa, con validez y fiabilidad como ventajas y las desventajas, sería dudosa (pág. 136). Es fundamental utilización de evaluación sumativa, evaluando y comparando a docentes del mismo nivel de experiencia, reconociendo la labor del profesional, en cualquier nivel educativo, pues el fin es brindar una educación de excelencia.

La visión del padre de familia, habla mucho de características del trabajo ideal que debería tener el docente del nivel inicial, en cuanto a su perfil. Por ejemplo, contrastando con la información científica, se puede decir que se mide la empatía, el fomento y desarrollo de la capacidad neta de investigación del niño de 4-5 años. Como le dice Gonzalez (2015): el docente además de ejercer la acción pedagógica, de hacer procesos que orienten y guíen aprendizajes, de hacer de mediador de la ruptura casa y su nuevo entorno educativo, de estimular el convivir permitiendo relaciones positivas, asertivas ya armónicas (pág. 1); dentro del ámbito educativo y familiar, debe guiar y orientar procesos, ya que para Fermín, González y Pazo (2009): todo esto está enfocado a un desarrollo integral del estudiante (pág. 27).

Se determina que se alcanza un nivel 4, pero con un promedio bajo, expresando que la calidad educativa percibida desde fuera solo llega a un $53 \%$ de los padres de familia, que son uno de los actores del sistema educativo más 
importantes. Por, lo tanto se tiene que trabajar de inmediato en la institución educativa, en un proceso de mejoramiento continuo que lleve a la excelencia ya a todos los decentes.

Se ha comprobado la hipótesis planteada, puesto que sí existe un desempeño de la labor docente, para la adquisición de aprendizajes, con calidad y calidez, de los docentes de Educación de nivel Inicial según los Estándares de Calidad Nacional.

\section{Referencias}

Arratia, A. (2010). Desempeño laboral y condiciones de trabajo docente en Chile: Influencias y percepciones desde los evaluados. Tesis. Chile: Universidad de Chile. Recuperado de:

http://www.tesis.uchile.cl/tesis/uchile/2010/cs-arratia a/pdfAmont/csarratia a.pdf

Castilla, F. (2014). La teoría del desarrollo cognitivo de Piaget aplicada en la clase de primaria. Trabajo de Grado. Segovia, España: Universidad de Valladolid. Recuperado de:

http://uvadoc.uva.es/bitstream/handle/10324/5844/TFG-

B.531. pdf? sequence $=1$ \&isAllowed $=y$

Dones, R. (2016). Serie “Los Precursores": María Montessori. Revista DONES, Tendencias y Enfoques. Recuperado de:

https://www.revistadones.com.ar/single-post/2016/11/28/Serie-LosPrecursores-Mar\%C3\%ADa-Montessori

Escobar, M. (2014a,b). Influencia de la interacción alumno-docente en el proceso enseñanza-aprendizaje. PAAKAT: Revista de Tecnología y Sociedad, 5(8), 1-8, e-ISSN: 2007-3607. Recuperado de:

http://www.udgvirtual.udg.mx/paakat/index.php/paakat/article/view/230

Espinoza, G. (2014). Desempeño docente en el proceso de enseñanzaaprendizaje en el nivel de Educación Básica Superior del Centro 
Educativo Colegio de Bachillerato Ciudad de Portovelo, del cantón Portovelo, Provincia de El Oro, en el año lectivo 2013-2014. Trabajo de fin de titulación. Ecuador: Universidad Técnica Particular de Loja. Recuperado de:

http://dspace.utpl.edu.ec/bitstream/123456789/11373/1/Espinosa Gal arza Gina Fabiola.pdf

Fermín, M., González, Y., \& Pazo, J. (2009). Formación del docente de educación inicial: consideraciones para atender el nivel maternal (período gestacional). Laurus, 15(29), 114-143, e-ISSN: 1315-883X. Recuperado de: http://www.redalyc.org/articulo.oa?id=76120642007

Gonzalez, M. (2015). Perfil de la educadora. Características personales. México: educacioninicial.com. [Página web]. Recuperado de:

http://www.educacioninicial.com/ei/contenidos/00/1900/1918.ASP

Hernández, U. (2017). La evaluación en el nuevo modelo educativo: un proceso paulatino. Gaceta de la política nacional de evaluación educativa, 3(8), 1-95. e-ISSN: 2448-5152. Recuperado de:

https://www.inee.edu.mx/wp-content/uploads/2018/12/G08ESP-1.pdf

INEE (2017). Directrices para mejorarla atención educativa de niñas, niños y adolescentes indígenas. México: Instituto Nacional para la Evaluación de la Educación - INEE. Recuperado de:

http://www.sev.gob.mx/servicio-profesionaldocente/files/2017/02/Directrices4.pdf

Ministerio de Educación Nacional (2013). Evaluación de competencias para el ascenso o reubicación de nivel salarial en el escalafón docente de los docentes y directivos docentes regidos por el decreto ley 1278 de 2002. Bogotá, Colombia: Ministerio de Educación Nacional de la República de Colombia. Recuperado de:

https://www.mineducacion.gov.co/proyectos/1737/articles$\underline{310888 \text { archivo pdf fisica.pdf }}$ 
Ministerio de Educación (2017a,b). Desempeño del Docente. Quito, Ecuador: Sistema Nacional de Evaluación del Ministerio de Educación. Recuperado de: https://educacion.gob.ec/desempeno-del-docente-sne/ Ministerio de Educación (2014). Currículo de Educación Inicial. ISBN: 9789942-07-625-0. Quito, Ecuador: Ministerio de Educación del Ecuador.

Morán-Barrios J. (2016). La evaluación del desempeño o de las competencias en la práctica clínica. $1^{\text {a }}$ Parte: principios y métodos, ventajas y desventajas. Educación Médica, 17(4), 130-139, e-ISSN: 1575-1813. Recuperado de:

http://dx.doi.org/10.1016/..edumed.2016.07.001

Ovalles, A. (2018). Necesidades de Capacitación de los Docentes de Educación Inicial sobre las Altas Capacidades Intelectuales. Revista Scientific, 3(10), 20-39, e-ISSN: 2542-2987. Recuperado de: https://doi.org/10.29394/Scientific.issn.2542-2987.2018.3.10.1.20-39

Robalino, M. (2005). ¿Actor o protagonista? Dilemas y responsabilidades sociales de la profesión docente. Revista PRELAC, (1), 7-23. Recuperado de: https://unesdoc.unesco.org/ark:/48223/pf0000144709

Soriano, A., \& Delgado, M. (2011). Buenas prácticas profesionales en la enseñanza de la educación física. Formación inicial y actualización. Un estudio exploratorio. Motricidad Humana, 12(1), 815, e-ISSN: 0718-9613. Recuperado de:

https://dialnet.unirioja.es/servlet/articulo?codigo=3706536

Tejedor, F. (2012a,b). Evaluación del Desempeño Docente. Revista Iberoamericana de Evaluación Educativa, 5(1e), 319-327, e-ISSN: 1989-0397. Recuperado de:

https://revistas.uam.es/index.php/riee/article/view/4447

Villarroel, P. (2015). Recorrido metodológico en educación inicial. Sophia, (19), 153-170, e-ISSN: 1390-8626. Recuperado de: 


\section{Mónica Alexandra Salto Cubillos}

e-mail: monica75salto@gmail.com

Nacida en Cuenca, Ecuador, el 4 de mayo del año 1975.

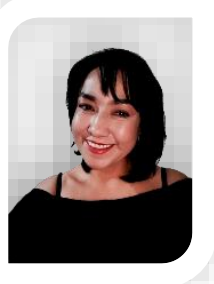

Licenciada en Educación General Básica; mejor graduada de la promoción año 2015 en la Universidad Estatal de Cuenca (U); Magister en Gerencia y Liderazgo Educacional de la Universidad Técnica Particular de Loja (UTPL); Docente de la Universidad Nacional de Educación (UNAE); Docente de Educación Inicial y en Educación General Básica. La línea de investigación a la cual inclino mis aportes, está orientada a temáticas del aprendizaje, en el nivel inicial principalmente por ser el nivel en el cual el niño(a) desarrolla todo su potencial cognitivo, emocional y social. Ponente y publicadora del artículo "Paradigmas Intrínsicos: la Neurodidáctica y la Felicidad". 


\section{Adrián Eduardo Cendón Sosa \\ e-mail: adricendon@hotmail.com}

Nacido en Cuenca, Ecuador, el 8 de diciembre del año

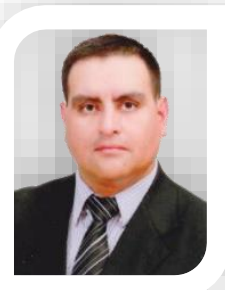
1980, de nacionalidad ecuatoriana y española. Ingeniero en Mecánica Automotriz; mejor graduado de la promoción año 2006 en la Universidad del Azuay (UDA); Magister en Administración de Empresas, de la Universidad de Especialidades Espíritu Santo (UEES); Educador en Metodología No Formal, Dirigente Scout IM4; Docente de Secundaria. Mi línea de investigación preferida, corresponde a temáticas del aprendizaje en el ciclo determinado desde la niñez hasta la juventud, para que las personas se desarrollen integralmente; Ponente y publicador del artículo "Paradigmas Intrínsicos: la Neurodidáctica y la Felicidad". 